Joel Gonçalves dos Santos joel.gonçalves.st@gmail.com

Fabiane Mondini fabiane.mondini@unesp.br. orcid.org/0000-0003-4975-6637 Universidade Estadual Paulista (UNESP), Instituto de Ciência e Tecnologia (ITC), Câmpus de Sorocaba, São Paulo, Brasil

\section{Um estudo sobre o tratamento formal dos números racionais}

\section{RESUMO}

O seguinte artigo tem por objetivo apresentar um estudo sobre o tratamento formal para alguns conceitos referentes aos números racionais, discutidos a partir de demonstrações. Nosso interesse pelo assunto pauta-se em nossa experiência vivida, enquanto professores e pesquisadores da área de Educação Matemática. Assumimos para este trabalho uma postura fenomenológica. Isso significa que nossas considerações não são generalidades, mas sim, interpretações e compreensões do que nos propomos a pesquisar. Justificamos a escrita do texto pela complexidade envolvendo a temática, visto que não há consenso entre educadores sobre quando iniciar, no contexto da sala de aula, o trabalho com a matemática formal. E, ao mesmo tempo, a escolha do tema justifica-se também por sua importância, visto que são as demonstrações matemáticas que fundamentam as teorias desta Ciência, garantindo sua validade ou não. Partimos pelos caminhos da História da Matemática, que nos mostra que, em princípio, as verdades da matemática eram constituídas de modo intuitivo, depois foram entrelaçadas pela Lógica Matemática e, na atualidade, são definidas por suas características lógico-epistemológicas, retóricas e heurísticas. Nesse contexto, destaca-se para nós os números racionais, pela riqueza matemática envolvida na constituição desse conjunto numérico e por sua presença na Educação Básica. Assim, nos debruçamos sobre o tratamento formal deste conjunto numérico e, enquanto educadores, não perdemos de vista o contexto educacional. Anunciamos que nossa intenção não é arbitrar sobre a formalização da matemática em seu ensino na Educação Básica, mas sim, expor um estudo teórico sobre algumas ideias matemáticas envolvendo os números racionais que sintetizam nossas compreensões.

PALAVRAS-CHAVE: Prova ou demonstração. Ensino de Matemática. Números racionais. 


\title{
INTRODUÇÃO
}

A importância da matemática para o avanço da Ciência e da Tecnologia é algo reconhecido desde o século XVIII. Tal reconhecimento impulsiona a inserção dessa ciência como uma disciplina do contexto escolar nos currículos de praticamente todos os países do mundo. Desde então, ela vem sendo abordada em diversas perspectivas, que a tematizam do ponto de vista de sua produção, de sua aplicabilidade, de seu ensino, de sua aprendizagem e de sua organização.

É remanescente do século XVIII também uma preocupação em possibilitar acesso a ideias avançadas da matemática aos estudantes dessa disciplina desde a educação básica, ou seja, de trabalhar a "Matemática Elementar de um Ponto de Vista Superior" (KLEIN, 2010). Dentre os caminhos para a inserção no contexto escolar das ideias avançadas da matemática, enquanto ciência, está a demonstração, que é um tipo de prova matemática.

Segundo Garnica (1995, p.12-13), a demonstração é

\begin{abstract}
uma explicação aceita por uma dada comunidade num dado momento, podendo ser rebatida, refutada ou aceita no interior da comunidade Matemática, porém, só são aceitas como provas as explicações que adotam uma forma particular, um conjunto de enunciados válidos organizados segundo certas regras, sendo que um enunciado ou é reconhecido como verdadeiro ou é deduzido a partir do precedente por regras de dedução válidas e pré-fixadas, do domínio da Lógica. A esse tipo particular de prova Balacheff chama demonstração.
\end{abstract}

De antemão, anunciamos que uma demonstração matemática pode ter muitos propósitos e significados. São as demonstrações, por exemplo, que estabelecem a validade relativa a um enunciado ou proposição, garantindo se é verdadeira ou falsa, bem como que nos convencem sobre uma verdade demonstrada (SILVA, 2002).

As demonstrações matemáticas podem ser pensadas como formas de argumentação dentro de uma teoria formal, pois não precisamos desenvolver toda uma discussão sobre uma afirmação matemática para que a sua validade seja aceita, uma vez que a própria demonstração da afirmação, desde que esteja de acordo com as regras da lógica, argumentará e convencerá aquele que estuda a prova em si (GARNICA, 2002).

Quando pensamos a demonstração nas aulas de Matemática da Educação Básica, o objetivo é a aprendizagem (HANNA 1995, p. 48). Segundo a autora, a demonstração possibilita a vivência do processo de descoberta, compreensões sobre o que é uma conjectura matemática, a experiência de formulação de conjecturas, o desenvolvimento do raciocínio matemático e a manipulação da escrita matemática.

As demonstrações matemáticas também nos auxiliam no desenvolvimento da capacidade de "explorar situações problemas, procurar regularidades, fazer e testar conjecturas, formular generalizações, pensar de maneira lógica" (MACHADO; SANTOS, 2011, p. 52).

Nesta perspectiva, o presente artigo tem por objetivo apresentar um estudo teórico sobre a demonstração de propriedades dos conceitos dos números racionais como uma possibilidade para a produção de conhecimento matemático. 
Partimos pelos caminhos da História da Matemática e nos debruçamos sobre o tratamento formal dos números racionais considerando o contexto da Educação Básica, apresentando ao leitor, um texto que discute este conjunto numérico, do ponto de vista formal.

\title{
PROCEDIMENTOS METODOLÓGICOS
}

Este estudo é de cunho qualitativo, desenvolvido de acordo com os princípios de uma postura fenomenológica. A opção pela Fenomenologia é porque a consideramos como uma forma radical de pensar.

\begin{abstract}
Ela parte, necessariamente, de caminhos conhecidos de fazerem-se as coisas, desafia os pressupostos aceitos e busca uma nova perspectiva para ver o fenômeno. Enquanto um método genuinamente radical fundamenta-se em novos conceitos, os quais, no começo, para aquele que nela se inicia, são estranhos e desconhecidos. À medida que se "habita" a nova linguagem, entende seus significados que se referem tanto às proposições, ideias e corpo de ideias (BICUDO, 1983, p.11)
\end{abstract}

Desse modo, nossas considerações não são generalidades, mas sim compreensões e interpretações acerca do que nos propomos a pesquisar. A pesquisa fenomenológica

está dirigida para significados, ou seja, para expressões claras sobre percepções que o sujeito tem daquilo que está sendo pesquisado, as quais são expressas pelo próprio sujeito que as percebe...)e não está interessado apenas nos dados coletados mas nos significados atribuídos pelos sujeitos entrevistados/observados (MARTINS; BICUDO, 1989, p.97)

Enquanto pesquisadores, temos a clareza da "não separação entre o percebido e a percepção de quem percebe, uma vez que é exigida uma correlação de sintonia, entendida como doação, no sentido de exposição, entre ambos" (BICUDO, 2012, p. 18).

Bicudo (1999) nos diz que percebemos mediante uma intencionalidade, pois enquanto pesquisadores, nos debruçamos sobre o fenômeno investigado, guiados pela consciência, compreendida como o estar voltada atentamente para algo que se mostra, que nos interessa. Quando algo está a mostra, ele não se mostra de imediato - depende a todo instante da intenção do sujeito que interroga, do rigor e da insistência para se obter aquilo que é característico, básico e essencial do fenômeno.

Sendo o fenômeno percebido pelo sujeito, a percepção é particular. Nesse sentido, a realidade já não será mais objetiva, mas se torna aquilo que se compreendeu, que se interpretou e que se comunicou do percebido. "É, portanto, perspectival, não havendo uma única realidade, mas tantas quantas forem suas interpretações e comunicações" (BICUDO, 1994, p. 18).

Assim, apresentamos uma compreensão possível: focamos o fenômeno demonstrações matemáticas no conjunto dos números racionais com a clareza da impossibilidade de esgotar o tema. generalizações de resultados passíveis de serem estendidos e aplicados a 
outros contextos. Entretanto, mediante o movimento específico da redução fenomenológica, as compreensões expressas apontam para as características nucleares ao fenômeno, [...] explicitando polissemias de significados de palavras e possíveis sentidos que elas carregam, e articulando em ideias cada vez mais abrangentes o expresso nos individuais (MONDINI; BICUDO, p. 03, 2019)

Assumir a fenomenologia como uma postura metodológica significa que procuramos compreender o fenômeno, partindo de como ele se mostra a nós, em nossa experiência vivida, ou seja, em disciplinas que tratam do tema no curso de Licenciatura em Matemática, em textos que versam sobre o assunto e em nossa prática enquanto professores de Matemática.

Os procedimentos são inseparáveis do fenômeno focado e do pesquisador. No entanto, "transcende a esfera da subjetividade do sujeito que põe em marcha a pesquisa" (MONDINI; BICUDO, 2019, p.03). Pois, de modo dialético, o movimento investigativo realiza ininterruptamente o pensar meditativo, articulando perguntas e revelando articulações clareadoras, que expomos na continuidade do texto.

\section{SOBRE SENTIDOS E SIGNIFICADOS DE DEMONSTRAÇÕES MATEMÁTICAS}

Segundo Bicudo (2002), as demonstrações matemáticas são artifícios utilizados por matemáticos para fundamentar uma teoria, pois são elas que garantem a validade ou não das afirmações matemáticas. Ainda, de acordo com o mesmo autor, os primeiros registros de demonstrações matemáticas que tivemos acesso são relativos aos séculos V e IV a.C., estão ligados ao conhecimento grego.

[...] os gregos herdaram - por meio de Tales - a matemática (geometria) dos egípcios. Mas, em algum ponto da história, no cadinho em que se formou o pensamento grego, em geral, e o pensamento matemático grego, em particular, uma faísca de genialidade alterou o material. Disso resultou não bastar mais ver para crer; para CRER era preciso PROVAR. O mundo sensível era mutável e ilusório. Havia mister de apreender o estável por trás da mudança, a unidade que se escondia na multiplicidade e o eterno no perecível (BICUDO, 1999, p. 118).

Num momento anterior, em civilizações como a dos egípcios e babilônios, não encontramos ideias semelhantes ao que hoje chamamos de uma demonstração matemática. Embora eles se valessem de instruções para a resolução de problemas, em suma, eram apenas passos recomendados, sem registros que justificassem os resultados obtidos. Tais resultados, em sua maioria, eram baseados nas experiências vividas, muitas vezes ligadas as necessidades cotidianas das sociedades.

Em relação à matemática egípcia e babilônica, Domingues (2002, p. 56) diz que

[...] nenhuma delas se baseou em qualquer estrutura axiomática que se pudesse servir de garantia para a validade dos procedimentos práticos de que essencialmente se compunham. O critério de confiabilidade das regras e procedimentos usados era simplesmente a concordância com a realidade a que se destinavam.

Domingues (2002) relata que os matemáticos egípcios utilizavam evidências físicas, métodos de tentativa e erro, comparações e em algumas situações, como 
no volume do tronco de pirâmides, deduções algébricas. Ainda, segundo o mesmo autor, é possível que os egípcios já tivessem a ideia de demonstração, mesmo que para isso não utilizassem nenhum formalismo.

A respeito da transição de uma matemática obtida apenas pelas observações da natureza ou por tentativa e erro, para uma matemática demonstrativa. Eves (2004, p.94) nos diz:

Pela primeira vez na matemática, como em outros campos, o homem começou a formular questões fundamentais como "Por que os ângulos da base de um triângulo isósceles são iguais?" e "Por que o diâmetro de um círculo divide esse círculo ao meio?". Os processos empíricos do Oriente antigo, suficiente o bastante para responder questões na forma de como, não mais bastavam para as indagações mais científicas na forma de porquê. Algumas experiências com o método demonstrativo foram se consubstanciando e se impondo, e a afeição dedutiva da matemática, considerada pelos doutos como sua característica fundamental, passou ao primeiro plano.

Outro fato importante aconteceu na Grécia Antiga, com o início do desenvolvimento da lógica, mais precisamente com Aristóteles (384- 322 a.C.) de Estagira, um grande sistematizador da lógica formal. $\mathrm{O}$ trabalho desenvolvido por ele traz em sua proposta o que chamamos de formas válidas de inferência.

Uma forma válida de inferência é um modo de se obter conclusões a partir de pressupostos; uma inferência é (logicamente) válida se a veracidade das conclusões depender apenas da veracidade dos pressupostos, bem como será formal se independer do conteúdo (do que é dito), mas depender da forma lógica das asserções (de como isso é dito) (SILVA, 2007, p. 49).

Silva (2007) utiliza o seguinte exemplo: "se assumo como premissa que todo homem é mortal e que Sócrates é um homem, segue que Sócrates é mortal" (SILVA, 2007, p. 49). Analisando essa frase percebemos que a validade de uma inferência não tem dependência em relação aos conceitos que aparecem na frase, como os conceitos de mortalidade e humanidade, tampouco com Sócrates.

A validade, como dita acima, é assumida a partir do que se disse. Entretanto, na lógica desenvolvida por Aristóteles, há uma estreita relação com as asserções envolvidas, e, por conseguinte, a sistematização da lógica "ocorre na sociedade grega quase contemporaneamente e de forma estreitamente relacionada com as profundas transformações que ocorriam na estruturação da própria língua grega" (MACHADO; SANTOS, 2001, p.78). Disso, os autores destacam que a lógica pouco se relacionava com a matemática na antiguidade, mas estava diretamente ligada ao idioma grego.

Porém, fica claro que todas as bases para uma matemática demonstrativa já estavam se desenvolvendo, mesmo que em suas fases iniciais. Até próximo ao século XIX, a demonstração era trabalhada com meios intuitivos e, segundo Domingues (2002, p. 61),

a partir de algum momento, porém, tornou-se necessário submeter a noção de demonstração a uma análise mais profunda, com vistas a reduzir o recurso ao uso da evidência intuitiva. Capítulos importantes da matemática, como o Cálculo, por exemplo, tinham sido explorados tão profundamente que a intuição apenas ou raciocínios heurísticos-geométricos já não bastavam para explicar alguns resultados aparentemente paradoxais. 
Entretanto, a lógica desenvolve-se ao longo do tempo e, a partir de um determinado momento da história - meados do século XIX - ela evolui: "privilegiando linguagens simbólicas e ampliando o seu repertório de modos válidos de inferência, ela foi capaz de fornecer um meio ideal de expressão e articulação para as teorias matemáticas, o que a lógica Aristotélica estava longe de poder prover" (SILVA, 2007, p.49). Dessa maneira, a lógica passa a ser parte fundamental nas teorias matemáticas. Mas como seria o processo e desenvolvimento de uma demonstração matemática?

De acordo com Bicudo, "demonstrar uma proposição (exprimindo uma propriedade de um conceito) significa argumentar pela aceitação de sua validade, a partir da validade de outras proposições já demonstradas" (BICUDO, 2002, p. 80). Porém, ainda segundo autor, existem algumas proposições que não são possíveis de serem demonstradas e, para essas, são estabelecidas algumas "arquiteturas das teorias matemáticas: que as consideram como sendo conceitos primitivos (axiomas) e conceitos derivados (teoremas)" (BICUDO, 2012, p. 80).

\footnotetext{
Um axioma ou postulado é uma sentença matemática que não é uma definição e não precisa ser demonstrada. Bem diferente do que ocorre com os teoremas - que precisam de demonstração -, os axiomas ou postulados não precisam ser demonstrados e são apresentados realmente como decretos. Entretanto, como as noções primitivas, esses "decretos" não surgem de opiniões pessoais isoladas, são frutos da experiência, da observação e, também, de certo "consenso coletivo" (MORAIS FILHO, 2012, p. 164).
}

"Um teorema é uma sentença matemática válida, cuja validade é garantida por uma demonstração matemática" (MORAIS FILHO, 2012, p. 164). Os livros de matemática apresentam sentenças que já foram provadas, ou seja, teoremas. Um teorema pode receber nomes distintos, dependendo do contexto em que ele está. Ele pode ser denominado de Corolário, Lema ou proposição.

\begin{abstract}
Chamamos Corolário a um teorema obtido como consequência de outro recém-provado. Nesse caso, o segundo teorema é chamado corolário do teorema provado. Já um teorema usado para provar outro que lhe sucede é chamado lema; podemos dizer que um lema é um teorema auxiliar ou preparatório, que será usado na demonstração de outro teorema. Em algumas ocasiões, chama-se proposição a um teorema que não é central no contexto e tem importância limitada (MORAIS FILHO, 2012, p. 137).
\end{abstract}

Assim, em matemática, todas as afirmações devem ser demonstradas, ou seja, provadas para serem válidas, a menos que sejam axiomas. Silva (2002) considera a demonstração matemática como sendo simultaneamente uma "peça de retórica destinada a convencer, como um encadeamento lógico conduzindo à verdade e ao conhecimento e como um possível catalisador de descoberta matemática" (SILVA, 2002, p. 68).

Nesse sentido, uma demonstração matemática é uma maneira de convencimento de uma verdade e ao mesmo tempo é um modo de produzir novas verdades, ou seja, como já enunciado por Bicudo (2002), é um modo de argumentar a partir de proposições já demonstradas, bem como um modo de originar esse encadeamento lógico. 
uma demonstração é realizada quando são apresentados argumentos, matematicamente válidos, para cada uma das afirmações que enunciaram, usaram factos conhecidos e anteriormente aceites como verdadeiros para bases das suas justificações (...), encadearam os argumentos uns nos outros de tal modo que uma ideia fluía da anterior sem deixarem "pontas soltas" ou contradições e deduziram, logicamente, uma conclusão. (BOAVIDA, 2001, p.13)

Para Silva (2002), as demonstrações matemáticas possuem como características os aspectos lógico-epistemológico, retórico e heurístico:

\footnotetext{
Chamarei de lógico-epistemológico esse aspecto das demonstrações que as mostra como objetos lógicos ideais, árvores ou sequências ordenadas no espaço lógico, segundo relações de dependência, ou consequência, lógica. E de retórico o aspecto das demonstrações, segundo o qual, elas aparecem como portadoras de força coercitiva de aquiescência às teses demonstradas. Há ainda um aspecto a ser considerado. Demonstrações podem ter também uma função heurística. Isto é, elas podem ser indutoras de descoberta matemática (SILVA, 2002, p. 69).
}

A partir desses aspectos observados, Silva (2002) refere-se a uma demonstração matematicamente perfeita como sendo uma junção desses fatores, ou seja, ela é "logicamente correta, compreensível a um agente racional com limitações cognitivas humanas, e, ainda assim, heuristicamente estimulante" (SILVA, 2002, p. 71). Assim entende-se que uma demonstração perfeita é aquela onde a riqueza heurística deve ser acessível a quem for se utilizar dela, levando em consideração que todos somos limitados cognitivamente, a única exigência é que ela esteja estruturada de acordo com as normas lógicas.

\section{O TRATAMENTO FORMAL DE ALGUMAS IDEIAS MATEMÁTICAS ENVOLVENDO NÚMEROS RACIONAIS}

Os números racionais são definidos como sendo os números da forma $\frac{a}{b}$, sendo $a$ e $b$ números inteiros e $b \neq 0$. O conjunto desses números é indicado pela letra $\mathbb{Q}$, e pode ser enunciado como:

$$
\mathbb{Q}=\left\{\frac{a}{b} \mid a, b \in \mathbb{Z}, b \neq 0\right\}
$$

em que $\mathbb{Z}$ indica o conjunto dos números inteiros:

$$
\mathbb{Z}=\{\ldots,-3,-2,-1,0,1,2,3, \ldots\} .
$$

Mas não é apenas na forma $\frac{a}{b}$, ou seja, por meio de uma fração, que um número racional pode ser representado. Podemos expressá-lo ainda em sua forma decimal, quando efetuamos a divisão do numerador $a$, da fração, pelo seu denominador $b$. Porém, a forma decimal de um número racional pode resultar ou em finita, ou em periódica, acontecendo a primeira todas as vezes em que temos um fator primo de 10 (2, 5 ou ambos) no denominador de uma fração, e apenas ele. Conforme demonstrado a seguir.

Teorema - Um número racional possui representação decimal finita se, e somente se, quando escrito na forma irredutível a decomposição em fatores primos de seu denominador possui apenas os fatores 2 ou 5 . 
Demonstração - Seja $V$ um número racional com uma quantidade de casas decimais finita, portanto $V=X+0, S_{1} S_{2} \ldots S_{n}$, em que $X \in \mathbb{Z}$ é a parte inteira, e $\mathrm{S}_{1} \mathrm{~S}_{2} \ldots \mathrm{S}_{\mathrm{n}}$, as casas decimais. Cada $\mathrm{Sj}$ é um número entre 0 e 9 . Notemos que, se $S_{j}=0$, para todo $\mathrm{j}=1,2, \ldots, n$, então $V=X$ é um número inteiro e podemos escrevê-lo na forma fracionaria como $\mathrm{V}=\frac{\mathrm{V}}{2^{0} 5^{0}}$.

Então, supondo que $\mathrm{Sj} \neq 0$, neste caso $0, \mathrm{~S}_{1} \mathrm{~S}_{2} \ldots \mathrm{S}_{\mathrm{n}} \times 10^{\mathrm{n}}=\mathrm{S}_{1} \mathrm{~S}_{2 \ldots} \mathrm{S}_{\mathrm{n}} \rightarrow$ $0, S_{1} S_{2} \ldots S_{n}=\frac{S_{1} S_{2} \ldots S_{n}}{10^{n}}$, e assim,

$$
\mathrm{V}=\mathrm{X}+\frac{\mathrm{S}_{1} \mathrm{~S}_{2} \ldots \mathrm{S}_{\mathrm{n}}}{10^{\mathrm{n}}} \rightarrow \mathrm{V}=\frac{\mathrm{X} \times 10^{\mathrm{n}}+\mathrm{S}_{1} \mathrm{~S}_{2} \ldots \mathrm{S}_{\mathrm{n}}}{2^{\mathrm{n} 5^{\mathrm{n}}}} .
$$

Dessa maneira, chegamos a uma representação fracionária de $V$ com numerador e denominador inteiros; logo $V$ é racional. Para se obter a forma irredutível da última fração acima, talvez seja necessário simplificar a quantidade de fatores primos comuns ao numerador e denominador, ficando apenas potências de 2 e 5 no denominador.

Reciprocamente, seja $\frac{\mathrm{n}}{\mathrm{m}}$ uma fração irredutível com $\mathrm{n} \in \mathbb{Z}, \mathrm{m}=2^{\mathrm{p}} 5^{\mathrm{q}} \in \mathbb{Z} \mathrm{e}$ $\mathrm{p}, \mathrm{q} \in \mathbb{Z}>0$. Supondo $\mathrm{p} \geq \mathrm{q}$, temos $\frac{\mathrm{n}}{\mathrm{m}}=\frac{\mathrm{n}}{2^{\mathrm{p}} 5^{\mathrm{q}}}=\frac{\mathrm{n}}{2^{\mathrm{p}} 5^{\mathrm{q}}} \times \frac{5^{\mathrm{p}-\mathrm{q}}}{5^{\mathrm{p}-\mathrm{q}}}=\frac{\mathrm{n} \times 5^{\mathrm{p}-\mathrm{q}}}{10^{\mathrm{p}}}$. Aqui, podemos concluir que $\frac{\mathrm{n}}{\mathrm{m}}$ possui $p$ casas decimais quando está nesta forma. Para o caso em que $\mathrm{p}<\mathrm{q}$ a demonstração é analoga, o que encerra a prova deste teorema.

Em algumas situações do contexto escolar, ao tematizar os números racionais é preciso fazer conversões matemáticas, tanto de frações para decimais, quanto de decimais para frações. Quando o número é dado na forma de fração, basta efetuar a divisão do numerador pelo denominador para obter o número decimal.

A conversão de decimal para fração, por sua vez, exige a classificação do decimal em finito ou dízima periódica, e o modo de proceder na conversão depende dessa classificação. Para o caso em que o decimal é finito, temos que seu denominador é uma potência de 10, como provado acima. Assim, para o converter em fração podemos pensar no seguinte exemplo: $0,64 \times 100=64 \rightarrow 0,64=$ $\frac{64}{100}$. Generalizando esta ideia para um decimal $P$ de $n$ casas, tem-se que $P=$ $c, d_{1} d_{2} \ldots d_{n}$, em que c é sua parte inteira e $d_{1} d_{2} \ldots d_{n}$ sua parte decimal. Ao multiplicarmos essa equação por $10^{n}$ teremos:

$$
10^{n} P=c P=c, d_{1} d_{2} \ldots d_{n} \rightarrow P=\frac{c P=c, d_{1} d_{2} \ldots d_{n}}{10^{n}} .
$$

O processo de conversão de um decimal periódico em fração se dá por meio de um processo de demonstração, ou prova matemática. Vejamos um exemplo: Temos que $0,2323232 \ldots=\frac{23}{99}$. Para provar a veracidade dessa asserção partimos da premissa de que existe um $x$, racional, tal que $x=0,232323 \ldots$ Se multiplicarmos ambos os lados dessa equação por 100, teremos $100 x=$ $23,2323 \ldots$ Sabemos que $23,23232 \ldots=23+x$, e, portanto, podemos escrever a equação $100 x=23+x$. Para encontrar a fração que gera o número decimal, basta calcular o valor de $x$, que no exemplo é $\frac{23}{99}$, como queríamos demonstrar.

Ainda, sobre os números racionais, podemos falar de suas propriedades, que são regras matemáticas que organizam a forma como operamos com esse conjunto de números. São propriedades dos números racionais: 
Adição em $\mathbb{Q}$, definição 1: Sejam a $=\frac{\mathrm{m}}{\mathrm{n}}$ e $\mathrm{s}=\frac{\mathrm{r}}{\mathrm{s}}$ elementos de $\mathbb{Q}$. Chama-se soma de a com b e indica-se por a + b o elemento de $\mathbb{Q}$ definido da seguinte maneira: $\mathrm{a}+\mathrm{b}=\frac{\mathrm{m}}{\mathrm{n}}+\frac{\mathrm{r}}{\mathrm{s}}=\frac{\mathrm{ms}+\mathrm{nr}}{\mathrm{ns}}$.

Mostremos inicialmente que a soma independe dos pares utilizados para definir a e b. De fato, se $\mathrm{a}=\frac{\mathrm{m}}{\mathrm{n}}=\frac{\mathrm{m} \prime}{\mathrm{n}^{\prime}}$ e $\mathrm{b}=\frac{\mathrm{r}}{\mathrm{s}}=\frac{\mathrm{r} \prime}{\mathrm{s}^{\prime}}$, então $\mathrm{mn}^{\prime}=\mathrm{nm}^{\prime}$ e $\mathrm{rs}^{\prime}=\mathrm{sr}^{\prime}$. Multiplicando a primeira dessas igualdades por $\mathrm{ss}^{\prime}$ e a segunda por $\mathrm{nn}^{\prime} \mathrm{e}$, em seguida, somando membro a membro as igualdades obtidas, têm-se $\mathrm{msn}^{\prime} \mathrm{s}^{\prime}+$ $r n s^{\prime} n^{\prime}=n s m^{\prime} s^{\prime}+n s r^{\prime} n^{\prime}, \quad(m s+r n) n^{\prime} s^{\prime}=n s\left(m^{\prime} s^{\prime}+r^{\prime} n^{\prime}\right), \quad$ o que garante $\frac{\mathrm{ms}+\mathrm{rn}}{\mathrm{ns}}=\frac{\mathrm{m}^{\prime} \mathrm{s}^{\prime}+\mathrm{r} / \mathrm{n} \prime}{\mathrm{n} / \mathrm{s}^{\prime}}$,

Portanto, a correspondência $(a, b) \rightarrow a+b$ quaisquer que sejam $a, b \in \mathbb{Q}$, é uma operação sobre $\mathbb{Q}$. Para essa operação, valem as seguintes propriedades, para quaisquer a, b, c $\in \mathbb{Q}$ :

$\left.A_{1}\right)(a+b)+c=a+(b+c)$ (associativa).

$\left.\mathbf{A}_{2}\right) \mathrm{a}+\mathrm{b}=\mathrm{b}+\mathrm{a}$ (comutativa).

$A_{3}$ ) Existe elemento neutro: é a classe de equivalência $\frac{0}{1}=\frac{0}{2}=\frac{0}{3} \ldots$, uma vez que $\frac{\mathrm{m}}{n}+\frac{0}{1}=\frac{\mathrm{m} \cdot 1+\mathrm{n} \cdot 0}{n \cdot 1}=\frac{m}{n}$,qualquer que seja $\frac{m}{n} \in \mathbb{Q}$. A classe de equivalência $\frac{0}{1}=\frac{0}{2}=\frac{0}{3} \ldots$ será denotada simplesmente por 0 .

$\mathrm{A}_{4}$ ) Para todo $\mathrm{a}=\frac{\mathrm{m}}{n} \in \mathbb{Q}$, existe um elemento $\mathrm{b}$ tal que $\mathrm{a}+\mathrm{b}=0$.

De fato, $\mathrm{b}=\frac{-\mathrm{m}}{n} \in \mathbb{Q}$, pois $\frac{\mathrm{m}}{n}+\frac{-\mathrm{m}}{n}=\frac{\mathrm{mn}+(-\mathrm{m}) \mathrm{n}}{n n}=\frac{0}{n}=0$. O elemento $\frac{-\mathrm{m}}{n}$ é chamado simétrico aditivo ou oposto de $\frac{\mathrm{m}}{n}$.

Multiplicação em $\mathbb{Q}$, definição 3: Chamamos produto de $\mathrm{a}=\frac{\mathrm{m}}{\mathrm{n}} \in \mathbb{Q}$ por $\mathrm{b}=$ $\frac{\mathrm{r}}{\mathrm{s}} \in \mathbb{Q}$ o elemento $\mathrm{ab}=\mathrm{a} \times \mathrm{b}=\frac{\mathrm{mn}}{\mathrm{rs}}$.

Como $\mathrm{mn} \in \mathbb{Z}$ porque $\mathrm{m}$ e $\mathrm{n}$ são inteiros e rs $\in \mathbb{Z}$ e considerando que $\mathrm{rs} \neq 0$, porque $r \neq 0$ e $s \neq 0$, então $\frac{\mathrm{mn}}{\mathrm{ns}} \in \mathbb{Q}$.

Logo, a lei que associa a cada $(\mathrm{a}, \mathrm{b}) \in \mathbb{Q} \times \mathbb{Q}$ seu produto ab $\in \mathbb{Q}$, ou seja, $(\mathrm{a}, \mathrm{b}) \rightarrow$ ab é uma operação sobre $\mathbb{Q}$ que recebe o nome de multiplicação.

Para a multiplicação em $\mathbb{Q}$, valem as seguintes propriedades:

$\left.\mathbf{M}_{1}\right)(\mathrm{ab}) \mathrm{c}=\mathrm{a}(\mathrm{bc})$ (associativa).

$\left.\mathbf{M}_{\mathbf{2}}\right) \mathrm{ab}=$ ba (comutativa).

$M_{3}$ ) Existe elemento neutro: é a classe de equivalência $\frac{1}{1}=\frac{2}{2}=\frac{3}{3}=\cdots$, uma vez que $\frac{\mathrm{m}}{n} \cdot \frac{1}{1}=\frac{\mathrm{m} \cdot 1}{n \cdot 1}=\frac{\mathrm{m}}{n}$, qualquer que seja $\frac{\mathrm{m}}{n} \in \mathbb{Q}$. A classe de equivalência $\frac{1}{1}=$ $\frac{2}{2}=\frac{3}{3}=\cdots$ será denotada simplesmente por 1 .

$\mathbf{M}_{4}$ ) Para todo $\mathrm{a}=\frac{\mathrm{m}}{\mathrm{n}} \in \mathbb{Q}^{*}$ existe um elemento $\mathrm{b}$, tal que $a b=1$.

Trata-se de $\mathrm{b}=\frac{n}{m}$, que é um elemento de $\mathbb{Q}$, pois $m \neq 0$, e $\frac{\mathrm{m}}{n} \cdot \frac{\mathrm{n}}{m}=\frac{\mathrm{mn}}{m n}=\frac{1}{1}=$ 1. O elemento $\frac{\mathrm{n}}{\mathrm{m}}$ é chamado simétrico multiplicativo ou inverso de $\frac{\mathrm{m}}{n} \mathrm{e}$ é denotado 
por $\frac{\mathrm{n}}{m}=\left(\frac{m}{n}\right)^{-1}$. Ou seja, todo a $\in \mathbb{Q}^{*}$ tem simétrico multiplicativo ou inverso. Ademais, é imediato que $\left(\left(\frac{m}{n}\right)^{-1}\right)^{-1}=\frac{m}{n}$.

Pode-se provar que o inverso de $\frac{m}{n} \in \mathbb{Q}^{*}$ é único. Isso posto, fica fácil provar que se $a, b \in \mathbb{Q}^{*}$, então $(a \cdot b)^{-1}=a^{-1} \cdot b^{-1}$. De fato:

$$
\begin{gathered}
(a \cdot b) \cdot\left(a^{-1} \cdot b^{-1}\right)=\left[(a \cdot b) \cdot a^{-1}\right] \cdot b^{-1}= \\
=\left[a \cdot\left(b \cdot a^{-1}\right)\right] \cdot b^{-1}=\left[a \cdot\left(a^{-1} \cdot b\right)\right] \cdot b^{-1}= \\
=\left[\left(a \cdot a^{-1}\right) \cdot b\right] \cdot b^{-1}= \\
=[1 \cdot b] \cdot b^{-1}=b \cdot b^{-1}=1 .
\end{gathered}
$$

A unicidade do inverso garante então que, de fato: $(a \cdot b)^{-1}=a^{-1} b^{-1}$.

D) $a(b+c)=a b+a c$.

Concluímos que um número racional pode ser escrito em forma de fração, ou em forma decimal (finita ou periódica). Todos os números que não possuem esta característica são denominados números não racionais. Quando, por exemplo, calculamos a raiz quadrada de 3 em uma calculadora científica, encontramos o valor de 1,732050808..., que em um olhar ingênuo pode ser classificado como uma dízima periódica. Porém, não é possível encontrar a dízima e nem seu fim, pois não existem. Esse fato é provado por meio de uma demonstração por absurdo.

No processo de demonstração por absurdo de uma "asserção $A$, supõe-se a falsidade de $\mathrm{A}$ e obtém-se como consequência uma falsidade qualquer ou, equivalentemente uma contradição. $O$ que mostra que $A$ não pode ser falsa, sendo, portanto, verdadeira" (SILVA, 2007, p. 52). Mas, como se daria a prova de que $\sqrt{3}$ é não racional?

Por meio de uma demonstração por absurdo e pela definição de número racional, começamos negando a asserção de que $\sqrt{3}$ é não racional. Logo, ele só pode ser racional. Se ele for um número racional, poderá ser escrito na forma $\frac{n}{m}=$ $\sqrt{3}$, com $m$ e $n$ primos entre si.

Elevando ambos os lados da equação ao quadrado, temos: $\frac{n^{2}}{m^{2}}=3 \rightarrow n^{2}=$ $3 m^{2}$. Logo $n^{2}$ é divisível por 3; e assim, $n$ também o é, ou seja, $n=3 r$, com $r$ inteiro. Disto e de $n^{2}=3 m^{2}$ segue-se que $3^{2} r^{2}=3 m^{2} \rightarrow 3 r^{2}=m^{2}$, mostrando que $m$ é divisível por 3 , o que é absurdo, pois $m$ e $n$ são primos entre si e sabemos, por definição, que não há um número que os divide simultaneamente, a não ser 1 .

\section{CONSIDERAÇÕES FINAIS}

No âmbito do ensino de matemática, as demonstrações podem favorecer aos estudantes o levantamento de hipóteses e de conjecturas, a resolução de problemas, a avaliação de estratégias e de resultados. Também podem favorecer a compreensão de que a matemática, assim como as demais ciências, é um conhecimento humano, em construção, fruto de saberes provenientes de diferentes culturas e épocas históricas. 
Entendemos que ensinar matemática a partir de um ponto de vista formal requer planejamento. Para inserir as demonstrações matemáticas no contexto escolar é preciso domínio dos procedimentos lógicos da linguagem matemática (símbolos, inferências lógicas e os métodos de demonstração). "A capacidade de compreender uma demonstração ou uma justificação teórica precisa de algum tempo, é certo, mas é algo que se pode desenvolver se houver intenção de o fazer e clareza e sensatez nos objetivos a atingir" (FREITAS, 2011, p. 8).

As demonstrações em matemática vêm nos trazer certeza acerca de uma determinada afirmação, ou asserção, que se faz a respeito dos objetos matemáticos - nesse texto, especificamente - sobre os números racionais: que são números fracionários, que se apresentam em sua forma irredutível e geram números decimais finitos ou decimais periódicos.

Apesar de a Matemática ser, por excelência, uma ciência hipotético- -
dedutiva, porque suas demonstrações se apoiam sobre um sistema de
axiomas e postulados, é de fundamental importância também considerar o
papel heurístico das experimentações na aprendizagem da Matemática
(BRASIL, 2017, p.267)

Ao apresentar as demonstrações ou provas matemáticas, como um possível caminho para subsidiar discussões sobre os números racionais, temos a intenção de promover um pensar sobre as potencialidades da inserção da linguagem formal no contexto escolar, sempre que possível e conveniente, de acordo com o planejado pelo professor de matemática.

É por meio da demonstração, por exemplo, que justificamos o porquê de números como a raiz quadrada de 3 não poder ser racional, apesar de em um primeiro olhar deixar essa impressão e, também, porque frações com denominadores com números primos 2 e 5 , ou ambos, se transformam em decimais finitos. 0 que é diferente de apenas dizer que eles são finitos por apresentarem os números 2 e 5 em seus denominadores. O raciocínio envolvido é outro. A demonstração envolve, segundo Davis e Hersh (1985), um modo de proceder formal, abstrato e simbólico, que exige conhecimento prévio e domínio da linguagem matemática utilizada. Há um consenso de que

\begin{abstract}
mesmo nos níveis elementar e médio é proveitoso desenvolver nos alunos o gosto pela argumentação em geral e pela demonstração como elemento central da própria matemática, como aliás vem referido de forma muito clara nos programas. Sem deduções, a matemática pode tornar-se uma simples colecção de resultados interessantes e úteis, mas desconexos, sem uma visão clara de quais são os pontos de partida e quais as conclusões que deles se podem tirar (FREITAS, 2011, p.1).
\end{abstract}

Quando há a compreensão de uma demonstração não se faz necessária a construção de discursos para que se aceitem as propriedades da teoria. É a teoria que traz as justificações e os convencimentos necessários à produção de conhecimento matemático. Ao inserir discussões que remetem às ideias de formalização no processo de ensinar matemática, é necessário dar ao estudante o acesso à linguagem formal e a um certo tipo de raciocínio, característico desta Ciência. 


\title{
A study on the formal treatment of rational numbers
}

\begin{abstract}
The objective of this paper is to present a study about the formal treatment of some concepts related to rational numbers, with discussions based on demonstrations. Our interest in the subject stems from own experience in the field of mathematics education both as teachers and researchers. For the present work, we assumed a phenomenological stance. This means that our considerations are not generalities, but interpretations and understandings of what we sought to study. The justification for writing this paper stems from the complexity of the theme, as there is no consensus among educators regarding when to start formal work on mathematics in a classroom context. The choice of theme is further justified by its importance, since these are mathematical demonstrations which underpin the theory of this science, thus ensuring its validity. We thread the path of the history of mathematics from the beginning, when mathematical truths were intuitively constituted, to be later intertwined by mathematical logic, to the present day, when they are defined by their logical-epistemological, rhetoric and heuristic characteristics. In this context, we were attracted to the study of rational numbers, due to the mathematical richness involved in the constitution of this numerical set, and their presence in elementary education. We focus on the formal treatment of rational numbers while, as educators, we try not to lose sight of the educational context. We state that it is not our intention to arbitrate on the formalization of mathematics or the teaching of this science in elementary education, but rather, we aim to introduce a theoretical study regarding some mathematical ideas involving rational numbers that synthesize our understandings.
\end{abstract}

KEYWORDS: Proof or demonstration. Teaching of mathematics. Rational numbers. 


\section{REFERÊNCIAS}

BICUDO, I. História da Matemática: o pensamento da filosofia grega antiga e seus reflexos na educação matemática do mundo ocidental. In: BICUDO, M. A. V. Pesquisa em Educação Matemática: concepções e perspectivas. São Paulo: Editora da UNESP, 1999.

BICUDO, I. Demonstração em matemática. BOLEMA: Boletim de Educação Matemática, Rio Claro, v. 15, n. 18, p. 79-90, 2002.

BICUDO, M. A. V. Prefácio. In: MARTINS, J.; BICUDO, M. A. V. (Org.). Estudos sobre existencialismo, fenomenologia e educação. 1. ed. São Paulo: Moraes, 1983.

BICUDO, M. A. V. Sobre a fenomenologia. In: BICUDO, M. A. V.; ESPÓSITO, V. H. C. Pesquisa qualitativa em educação. Piracicaba: UNIMEP, 1994. Disponível em: http://www.mariabicudo.com.br/resources/CAPITULOS_DE_LIVROS/Sobre\%20a \%20fenomenologia.pdf. Acesso em: 02 mar. 2019.

BICUDO, M. A. V. Contribuição da fenomenologia para a educação. In: BICUDO, M. A. V.; CAPPELLETTI, I. F. (Orgs). Fenomenologia: uma visão abrangente da educação. São Paulo: Olho d’Água, p. 11-51, 1999.

BICUDO, M. A. V. A pesquisa em educação matemática: a prevalência da abordagem qualitativa. Revista Brasileira de Ensino de Ciência e Tecnologia, Ponta Grossa, v. 5, n. 2, 2012. Disponível em:

http://www.mariabicudo.com.br/resources/ARTIGOS/A\%20pesquisa\%20em\%20e duca\%C3\%A7\%C3\%A30\%20matem\%C3\%A1tica\%20a\%20preval\%C3\%AAncia\%20 da\%20abordagem\%20qualitativa.pdf. Acesso em: 02 mar. 2019.

BOAVIDA, A. M. Um olhar sobre o ensino da demonstração em Matemática. Revista Educação e Matemática, n. 63, p. 11-15, 2001. Disponível em: https://www.researchgate.net/publication/281778076_DEMONSTRACOES_MATE MATICAS_NA_EDUCACAO_BASICA_COM_A_PALAVRA_OS_PROFESSORES_DE_M ATEMATICA. Acesso em: 20 fev. 2020.

BRASIL. Lei no 13.415, de 16 de fevereiro de 2017, Diário Oficial da União, Brasília, 17 de fevereiro de 2017. Disponível em:

http://basenacionalcomum.mec.gov.br/images/BNCC_El_EF_110518_versaofinal _site.pdf. Acesso em: 02 fev. 2020.

DAVIS, F. J. e HERSH, R. A experiência matemática. Tradução de João Bosco Pitombeira. Rio de Janeiro: F. Alves, 1985

DOMINGUES, H. H. A demonstração ao longo dos séculos. BOLEMA: Boletim de Educação Matemática, v. 15, n.18, p. 55-67, 2002.

EVES, H. Introdução à história da matemática, tradução de Hygino $\mathrm{H}$. Domingues, v. 2, 2004. 
FREITAS, P. J. A demonstração matemática no ensino básico e secundário. Atas do ProfMat 2011, p. 1-12, 2011. Disponível em: https://www.profmatsbm.org.br/wp-ontent/uploads/sites/23/2016/08/Sumario_PROFMAT_2011.pdf. Acesso em: 20 fev. 2020.

GARNICA, A. V. M. Fascínio da técnica, declínio da crítica: Um estudo sobre a prova rigorosa na formação do professor de Matemática. Tese (Doutorado em Educação Matemática) - IGCE-UNESP, Rio Claro, 1995.

GARNICA, A. V. M. As demonstrações em educação matemática: um ensaio. BOLEMA: Boletim de Educação Matemática, v. 15, n. 18, p. 73-81, 2002.

HANNA, G. Challenges to the importance of proof. For the learming of mathematics, 1995, n.15. p. 42-49.

KLEIN, F. Elementary Mathematics from an Advanced Standpoint: Aritmetics, Algebra, Analysis. USA: Breinigsville. 2010.

MACHADO, S.; SANTOS, L. Demonstração matemática no 8o ano no contexto de utilização do geometer's sketchpad. Revista de Educação, v. 18, n. 1, p. 49-82, 2011. Disponível em:

https://repositorio.ul.pt/bitstream/10451/4881/1/Machado\%20\%26\%20Santos\% 20(2011).pdf. Acesso em: 20 fev. 2019.

MARTINS, J.; BICUDO, M. A. V. A pesquisa qualitativa em Psicologia. 1. ed. São Paulo: Editora Moraes, 1989.

MORAIS FILHO, D. C. Um convite à Matemática: Com técnicas de demonstração e notas históricas. SBN. 2012.

MONDINI, F.; BICUDO, M. A. V. Uma interpretação analítica da organização escolar da Matemática durante a Primeira República Brasileira. Zetetike. v. 27, 2019. e019032. Disponível em:

https://periodicos.sbu.unicamp.br/ojs/index.php/zetetike/issue/view/1589.

Acesso em: 18 jun. 2020.

SILVA, J. J. Demonstração Matemática da Perspectiva da Lógica

Matemática. BOLEMA: Boletim de Educação Matemática, v. 15, n. 18, p. 68-78, 2002.

SILVA, J. J. Filosofias da matemática. Unesp, 2007. 
Recebido: 19 nov. 2019

Aprovado: 04 mai. 2020

DOI: 10.3895/actio.v5n2.11300

Como citar:

SANTOS, J. G. dos; MONDINI, F. Um estudo sobre o tratamento formal dos números racionais. ACTIO,

Curitiba, v. 5, n. 2, p. 1-16, mai./ago. 2020. Disponível em: <https://periodicos.utfpr.edu.br/actio>. Acesso em:

$\mathrm{XXX}$

Correspondência:

Joel Gonçalves dos Santos. Rua das Três Marias, n. 198. Parque Novo Santo Amaro. São Paulo, São Paulo, Brasil.

Direito autoral: Este artigo está licenciado sob os termos da Licença Creative Commons-Atribuição 4.0 Internacional. 